\title{
Bacterial quorum sensing compounds are important modulators of microbe-plant interactions
}

\author{
Anton Hartmann ${ }^{1 *}$, Michael Rothballer ${ }^{1}$, Burkhard A. Hense ${ }^{2}$ and Peter Schröder ${ }^{1}$ \\ ${ }^{1}$ Research Unit Microbe-Plant Interactions, Department of Environmental Sciences, Helmholtz Zentrum München, German Research Center for Environmental \\ Health $(\mathrm{GmbH})$, Neuherberg, Germany \\ 2 Institute of Computational Biology, Helmholtz Zentrum München, German Research Center for Environmental Health (GmbH), Neuherberg, Germany \\ *Correspondence: anton.hartmann@helmholtz-muenchen.de
}

Edited by:

Gabriele Berg, Graz University of Technology, Austria

Reviewed by:

Ingyu Hwang, Seoul National University, South Korea

Keywords: Quorum sensing, $\mathrm{N}$-acyl-homoserine lactones, systemic responses, innate immunity, mutualists, endophytic bacteria

\section{PLANT-MICROBIOME INTERACTIONS IN THE LIGHT OF THE HOLOBIONTIC CONCEPT}

Higher organisms evolved in the omnipresence of microbes, which could be of pathogenic or symbiotic nature. A framework of response patterns evolved which is known as innate immunity. A major part of this response is the recognition of microbial-associated molecular patterns (MAMP) such as chitin or lipochitooligosaccharides, peptidoglycan, lipopolysaccharides or flagellum structures, and the initiation of efficient plant defence reactions (Janeway and Medzhitov, 2002; Jones and Dangl, 2006). However, there are many plantassociated endophytic bacteria known, which are living within plants without triggering persistent and apparent defence responses or visibly do not harm the plant. In some cases, even a stimulation of plant growth due to the presence of specific players within the plant microbiome was reported (Turner et al., 2013). It is now generally accepted, that plant performance and activities can only be characterized and understood completely, if the "holobiont," the plant plus the intimately associated microbiota, is considered (Zilber-Rosenberg and Rosenberg, 2008). The evolutionary advantage of an integrated holobiontic system is characterized by a much better adaptability and flexibility towards rapidly changing adverse environmental conditions. It is still mostly unknown, which particular plant genetic loci are controlling the interactions with the plant microbiome and which signals are steering this cooperativity. Mutualistic microbes are able to overcome or short-circuit plant defence responses to enable successful colonization of the host (Zamioudis and Pieterse, 2012; Alqueres et al., 2013). Beneficial associations with microbes other than mycorhiza or Rhizobia are also controlled by systemically regulated or autoregulated processes on top of the basic innate immunity response. The induction of systemic immunity responses like ISR (induced systemic resistance) by some beneficial rhizosphere bacteria or the SAR (systemic acquired resistance) response provoked by pathogens are results of multiple response cascades employed by the plant host to respond to microbial and other environmental interactions. However, the entire response network is by far not yet revealed. For example, bacteria-induced plant responses resulting in improved resistance towards pathogens can also be due to the perception of secondary metabolites, like the surfactin lipopeptide, produced by certain biocontrol Bacilli (Garcia-Gutiérrez et al., 2013) or volatile compounds of plant-associated microbes (Yi et al., 2010). The biocontrol activity of microbial inoculants is probably due to multiple effects of their secondary metabolites to achieve direct inhibition of the pathogenic counterpart as well as an increase of systemic resistance of the plant host.

\section{BACTERIAL QUORUM SENSING MOLECULES LIKE $\mathrm{N}$-acyl HOMOSERINE LACTONES MODULATE PLANT RESPONSES TOWARD CONTACT WITH BACTERIA}

It is hypothesized, that eukaryotic organisms developed ways to sense microbes in addition to the recognition of their MAMPs by their diffusible small molecules. A very ancient and basic feature of unicellular bacteria is their way of environmental sensing and social communication. In many Gram-negative bacteria the synthesis of autoinducers of the $\mathrm{N}$ acyl-homoserine lactone (AHL) type is tightly regulated in response to cell density or the cell "quorum" (Eberl, 1999). These metabolites are released into the cellular environment to sense the quality of the ecological niche in terms of diffusion space and the density and distribution of their own population. This environmental sensing mechanism helps to adapt the regulation of their gene expression to the given conditions in their habitat and thus optimizes the fitness of the population. Therefore, the generally known term "quorum sensing" (QS) was supplemented by the more broadly defined concept of "efficiency sensing" (Hense et al., 2007). Since this optimization of in situ gene expression is of very basic importance, autoinducer QS-molecules are widespread among bacteria and have quite different molecular structures. AHL are common in Gram-negative bacteria, while cyclic peptides as QS-signals are only to be found in Gram-positive bacteria. The detailed structure of the AHL-molecules can vary; the acyl side chain consists of 4-14 carbon atoms and may also contain double bonds. The C3-atom can be hydroxylated or oxidized to a carbonyl-carbon; thus, considerable information and quite different physicochemical properties can be present within these different AHLstructures. As is outlined below, also plants have obviously learned during their evolution to respond to these QS compounds in 
Table 1 | Recent findings of direct AHL impact on different plants.

\begin{tabular}{|c|c|c|c|}
\hline AHL type & Plant reaction & Plant species & References \\
\hline Short chain length & Increased transpiration, stomatal conductance & Phaseolus vulgaris & Joseph and Phillips, 2003 \\
\hline C6 & Primary root elongation & A. thaliana & von Rad et al., 2008 \\
\hline C6 & $\begin{array}{l}\text { Upregulation of metabolism, transport and } \\
\text { transcriptional regulation }\end{array}$ & A. thaliana & von Rad et al., 2008 \\
\hline C6 (Serratia liquefaciens) & Upregulation of defense genes & Lycopersicon esculentum & Schuhegger et al., 2006 \\
\hline $\mathrm{C} 6, \mathrm{C} 8, \mathrm{C} 10$ & Lactonase induction & Pachyrhizus erosus & Götz et al., 2007 \\
\hline Oxo-C6, oxo-C8 & G-protein coupled receptors for root growth & A. thaliana & Jin et al., 2012; Liu et al., 2012 \\
\hline 3-oxo-C6 (Serratia plymuthica) & Triggering plant immunity & $\begin{array}{l}\text { Cucumis sativa } \\
\text { Lycopersicon esculentum }\end{array}$ & Pang et al., 2009 \\
\hline $\mathrm{C} 6, \mathrm{C} 8, \mathrm{C} 10$ & Root and shoot growth & Hordeum vulgare & Götz et al., 2007 \\
\hline $3-\mathrm{O}-\mathrm{C} 10$ & Adventitious root formation & Vigna radiata & Bai et al., 2012 \\
\hline C10 & Lateral root formation & A. thaliana & Bai et al., 2012 \\
\hline C12 & Root hair development & A. thaliana & Ortíz-Castro et al., 2008 \\
\hline 3-oxo-C12 from $P$. aeruginosa & $\begin{array}{l}\text { Defense and stress management genes, } \\
\text { phytohormones, and metabolic regulation }\end{array}$ & Medicago truncatula & Mathesius et al., 2003 \\
\hline oxo-C12 & Resistance induction & A. thaliana & Schikora et al., 2011 \\
\hline oxo-C14 & Systemic resistance against Golovinomyces orontii & A. thaliana & Schikora et al., 2011 \\
\hline oxo-C14 & Systemic resistance against Blumeria graminis & Hordeum vulgare & Schikora et al., 2011 \\
\hline $\begin{array}{l}\text { 3-oxo-C16 from } \\
\text { (Sinorhizobium meliloti) }\end{array}$ & $\begin{array}{l}\text { Defense and stress management genes, } \\
\text { phytohormones and metabolic regulation }\end{array}$ & Medicago truncatula & Mathesius et al., 2003 \\
\hline
\end{tabular}

different specific ways. We speculate, that QS-compounds are early signals indicating that pathogens are in the surroundings to gather themselves for attack or that mutualists are about to interact with roots.

The first demonstration of specific responses of a plant to bacterial AHLs was demonstrated for the legumes Phaseolus vulgaris (Joseph and Phillips, 2003) and Medicago truncatula (Mathesius et al., 2003) (Table 1). AHLs from symbiotic (Sinorhizobium meliloti) or pathogenic (Pseudomonas aeruginosa) bacteria provoked at concentrations as low as nanoto micromolar significant changes in the accumulation of over 150 proteins. Auxinresponsive and flavonoid synthesis proteins were induced and also a secretion of plant metabolites that mimic QS compounds were found, which may have the potential to disrupt QS signaling by associated bacteria. In tomato plants, a specific induction of systemic resistance proteins after inoculation of the roots with C4- and C6-side chain AHL-producing Serratia liquefaciens MG1 was discovered independently (Hartmann et al., 2004; Schuhegger et al., 2006). The fungal leaf pathogen Alternaria alternata was much less effective, when $S$. liquefaciens MG 1 wild type had been inoculated to roots of tomato plants as compared to the
AHL-negative mutant. It could be shown, that salicylic acid was increased as well as SA- and ethylene-dependent defence genes (i.e., PR1a) in MG1-inoculated plants. Furthermore, Serratia plymuthica HROC48, producing C4-/C6- and OHC4/OHC6-homoserine lactones, is able to induce ISR-like systemic protection of bean and tomato plants against the fungal leaf pathogen Botrytis cinnera; this response was greatly reduced with mutants impaired in AHL-production (Liu et al., 2007; Pang et al., 2009). In contrast, Arabidopsis thaliana responds to short (C4- and C6-) $N$-acyl AHL-compounds in a different manner: C4- and C6- homoserine lactones alter the expression of selected hormonal regulated genes which results in changes of the plant's hormone content, in particular an increased auxin/cytokinin ratio (von Rad et al., 2008). However, no systemic resistance response was found to be induced in $A$. thaliana when roots were stimulated with short side-chain AHLs. Ortíz-Castro et al. (2008) found that C10-homoserine lactone elicited developmental changes in the root system in Arabidopsis plants by altering the expression of cell division and differentiationrelated genes. Furthermore, Liu et al. (2012) and Jin et al. (2012) demonstrated that the root stimulatory effect of C6- and
C8- homoserine lactones in Arabidopsis plants is mediated through the G-protein coupled receptor encoded by AtGPA1. In mung bean, oxoC10-homoserine lactone activates auxin-induced adventitious root formation via $\mathrm{H}_{2} \mathrm{O}_{2}$ - and $\mathrm{NO}$-dependent cyclic GMP signaling (Bai et al., 2012). On the other hand, $N$-acyl-AHLs with $\mathrm{C} 12$ and C14- side chains induce systemic resistance to the obligate biotrophic fungus Golovinomyces orontii in A. thaliana and to Blumeria graminis $\mathrm{f}$. sp. hordei in barley (Hordeum vulgare) (Schikora et al., 2011). This response is mediated through altered activation of AtMPK6. The mitogenactivated protein kinases AtMPK3 and AtMPK6 were stronger activated by the model elicitor flg22 in the presence of C12or C14-AHL compounds which resulted in a higher expression of the defencerelated transcription factors WRKY26 and WRKY29 as well as the PR1 gene (Schikora et al., 2011). Thus, AHLs with short and medium side chain lengths induce developmental effects on root architecture, while long side chain AHLs induce systemic resistance in A. thaliana (Schenk et al., 2012). Furthermore, it was shown, that better water soluble short side chain AHL-compounds are actively taken up into plant roots and transported along the roots into the shoot; in contrast, the 
lipophilic long acyl side chain AHLs are not transported in barley and A. thaliana. (Götz et al., 2007; von Rad et al., 2008; Sieper et al., 2014). However, no uptake was detected in the legume yam bean (Pachyrhizus erosus (L.) Urban) (Götz et al., 2007). The latter finding corroborates the report of Delalande et al. (2005) that legumes like Lotus corniculatus produce lactonases which degrade AHLs and prevent their uptake and transport. In barley, it could further be demonstrated that C8- and C10-AHLs are taken up in a cell energy dependent manner by ABCtransporters into the root and transported via the central cylinder into the shoot (Sieper et al., 2014).

Interestingly, several plants have been demonstrated to produce AHL-mimic substances or to develop other activities influencing QS of plant associated bacteria (Gao et al., 2003; Bauer and Mathesius, 2004). Flavonoids released by legumes increase the expression of AHL synthesis genes in Rhizobia (Pérez-Montano et al., 2011). Indole acetic acid and cytokinin biosynthesis of Gypsophila was shown to influence QS, type III secretion system and gall formation activity by Pantoea plantarum (Chalupowicz et al., 2009). On the other hand, tobacco plants have been engineered to produce short- and long-side chain AHL-compounds which could be detected in substantial amounts at leaf and root surfaces as well as in soil (Scott et al., 2006). Constitutive expression of QS genes in transgenic tobacco plants leads to alteration in induced systemic resistance elicited by the rhizobacterium Serratia marcescens 90-166 (Ryu et al., 2013). Furthermore, transgenic tomato plants engineered to produce different AHL-compounds were demonstrated to alter the activity of plant growth promoting rhizobacteria and resulted, e.g., in increased salt tolerance (Barriuso et al., 2008). We hypothesize, that QS in a plant-microbe holobiont system should be regarded in a bidirectional way with influences from the plant and the microbial partners.

Uptake of AHL-compounds and specific perception of AHLs in animal cells were also studied intensively in recent years (Teplitski et al., 2011; Hartmann and Schikora, 2012). 3-oxoC12-homoserine lactone (C12-AHL), the major AHL-compound of Pseudomonas aeruginosa, was shown to selectively impair the regulation of the nuclear transcription factor NF- $\kappa$ B which controls innate immune responses in mammalian cells (Kravchenko et al., 2008). C12AHL also impaired human dendritic cell functions required for priming of T-cells (Bernatowicz et al., submitted). Since the response to AHL-compounds in mammalian systems is complicated due to the interferences with the adaptive immune system, plants provide an ideal model for the detailed interaction studies of basic innate immune responses and developmental processes with $\mathrm{N}$-acylhomoserine lactones as modifying bacterial effector molecules.

\section{REFERENCES}

Alqueres, S., Menses, C., Rouws, L., Rothballer, M., Baldani, I., Schmid, M., et al. (2013). The bacterial superoxide dismutase and glutathione reductase are crucial for endophytic colonization of rice roots by Gluconacetobacter diazotrophicus strain PAL5. Mol. Plant-Microbe Interact. 26, 937-945. doi: 10.1094/MPMI-12-12-0286-R

Bai, X., Todd, C. D., Desikan, R., Yang, Y., and Hu, X. (2012). N-3-oxo-decanoyl-L-homoserinelactone activates auxin-induced adventitious root formation via hydrogen peroxide- and nitric oxide-dependent cyclic GMP signaling in mung bean. Plant Physiol. 158, 725-736. doi: 10.1104/pp.111.185769

Barriuso, J., Solano, B. R., Fray, R. G., Cámara, M., Hartmann, A., and Gutierrez Manero, F. J. (2008). Transgenic tomato plants alter quorum sensing in plant growth-promoting rhizobacteria. Plant Biotech. J. 6, 442-452. doi: 10.1111/j.14677652.2008.00331.x

Bauer, W. D., and Mathesius, U. (2004). Plant responses to bacterial quorum sensing signals. Curr. Opin. Plant Biol. 7, 429-433. doi: 10.1016/j.pbi.2004.05.008

Chalupowicz, L., Barash, I., Panijel, M., Sessa, G., and Manulis-Sasson, S. (2009). Regulatory interaction between quorum-sensing, auxin, cytokinin, and the hrp regulon in relation to gall formation and epiphytic fitness of Pantoea agglomerans pv. Gypsophilae. Mol. Plant Microbe Interact. 22, 849-856. doi: 10.1094/MPMI-227-0849

Delalande, L., Faure, D., Raffoux, A., Uroz, S., D'Angelo-Picard, C., Elasri, M., et al. (2005). $\mathrm{N}$-hexanoyl-L-homoserine lactone, a mediator of bacterial quorum sensing regulation, exhibits plant-dependent. FEMS Microbiol. Ecol. 52, 13-50.

Eberl, L. (1999). N-acyl-homoserine lactonemediated gene regulation in Gram-negative bacteria. Syst. Appl. Microbiol. 22, 493-506. doi: 10.1016/S0723-2020(99)80001-0

Gao, M., Teplitski, M., Robinson, J. B., and Bauer, W. D. (2003). Production of substances by Medicago truncatula that affect bacterial quorum sensing.
Mol. Plant Microbe Interact. 16, 827-834. doi: 10.1094/MPMI.2003.16.9.827

Garcia-Gutiérrez, L., Zeriouh, H., Romero, D., Cubero, J., de Vicente, A., and Pérez-Garcia A. (2013). The antagonistic strain Bacillus subtilis UMAF6639 also confers protection to melon plants against cucurbit powdery mildew by activation of jasmonate- and salicylic acid-dependent defence response. Microb. Biotechnol. 6, 264-274. doi: 10.1111/1751-7915.12028

Götz, C., Fekete, A., Gebefügi, I., Forczek, S., Fuksová, K., Li, X., et al. (2007). Uptake, degradation and chiral discrimination of $N$-acyl-D/L-homoserine lactones by barley (Hordeum vulgare) and yam bean (Pachyrhizus erosus) plants. Anal. Bioanal. Chem. 389, 1447-1457. doi: 10.1007/s00216-0071579-2

Hartmann, A., Gantner, S., Schuhegger, R., Steidle, A., Dürr, C., Schmid, M., et al. (2004). "N-acylhomoserine lactones of rhizosphere bacteria trigger systemic resistance in tomato plants" in Biology of Molecular Plant-Microbe Interactions, Vol. 4, eds B. Lugtenberg, I. Tikhonovich, and N. Provorov (St. Paul, Minnesota: MPMI-Press), 554-556.

Hartmann, A., and Schikora, A. (2012). Quorum sensing of bacteria and trans-kingdom interactions of $N$-acyl homoserine lactones with eukaryotes J. Chem. Ecol. 38, 704-713. doi: 10.1007/s10886012-0141-7

Hense, B. A., Kuttler, C., Müller, J., Rothballer, M., Hartmann, A., and Kreft, J.-U. (2007). Does efficiency sensing unify diffusion and quorum sensing? Nat. Rev. Microbiol. 5, 230-239. doi: 10.1038/nrmicro1600

Janeway, C. A. Jr., and Medzhitov, R. (2002) Innate immune recognition. Annu. Rev. Immunol. 20, 197-216. doi: 10.1146/annurev.immunol.20. 083001.084359

Jin, G., Liu, F., Ma, H., Hao, S., Zhao, Q., Bian, Z., et al. (2012). Two G-protein-coupled-receptor candidates, Cand 2 and Cand7, are involved in Arabidopsis root growth mediated by the bacterial quorum-sensing signals $N$-acyl- homoserine lactones. Biochem. Biophys. Res. Commun. 417, 991-995. doi: 10.1016/j.bbrc.2011.12.066

Jones, J. D. G., and Dangl, J. L. (2006). The plant immune system. Nature 444, 323-329. doi: 10.1038/nature05286

Joseph, C. M., and Phillips, D. A. (2003). Metabolites from soil bacteria affect plant water relations. Plant Physiol. Biochem. 41, 189-192. doi: 10.1016/S09819428(02)00021-9

Kravchenko, V. V., Kaufmann, G. F., Mathison, J. C., Scott, D. A., Katz, A. Z., Wood, M. R., et al. (2008). Modulation of gene expression via disruption of NF- $\kappa \mathrm{B}$ signalling by a bacterial small molecule. Science 321, 259-263. doi: 10.1126/ science. 1156499

Liu, F., Bian, Z., Jia, Z., Zhao, Q., and Song, S. (2012). The GCR1 and GPA1 participate in promotion of Arabidopsis primary root elongation induced by $\mathrm{N}$-acyl-homoserine lactones, the bacterial quorum-sensing system. Mol. Plant Microbe Interact. 25, 677-683. doi: 10.1094/MPMI-1011-0274

Liu, X., Bimerew, M., Ma, Y., Müller, H., Ovadis, M., Eberl, L., et al. (2007). Quorum-sensing signaling is required for production of the antobiotic pyrrolnitrin in a rhizospheric biocontrol strain of 
Serratia plymuthica. FEMS Microbiol. Lett. 270, 299-305. doi: 10.1111/j.1574-6968.2007.00681.x

Mathesius, U., Mulders, S., Gao, M., Teplitski, M., Caetano-Anoliés, G., Rolfe, B. G., et al. (2003). Extensive and specific responses of a eukaryote to bacterial quorum sensing signals. Proc. Natl. Acad. Sci. U.S.A 100, 1444-1449. doi: 10.1073/pnas.262672599

Ortíz-Castro, R., Martinez-Trujillo, M., and LópezBucio, J. (2008). $N$-acyl-homoserine lactones: a class of bacterial quorum-sensing signals alter post-embryonic root development in Arabidopsis thaliana. Plant Cell Environ. 31, 1497-1509. doi: 10.1111/j.1365-3040.2008.01863.x

Pang, Y., Liu, X., Ma, Y., Chernin, L., Berg, G., and Gao, K. (2009). Induction of systemic resistance, root colonisation and biocontrol activities of the rhizospheric strain of Serratia plymuthica are dependent on $N$-acyl homoserine lactones. Eur. J. Plant Pathol. 124, 261-268. doi: 10.1007/s10658008-9411-1

Pérez-Montano, F., Guasch-Vidal, B., GonzálezBarroso, S., López-Baena, F. J., Cubo, T., Ollero, F. J., et al. (2011). Nodulation-gene-inducing flavonoids increase overall production of autoinducers and expression of $\mathrm{N}$-acyl-homoserine lactone synthesis genes in Rhizobia. Res. Microbiol. 162, 715-723. doi: 10.1016/j.resmic.2011. 05.002

Ryu, C.-M., Choi, H. K., Lee, C.-H., Murphy, J. F., Lee, J.-K., and Kloepper, J. W. (2013). Modulation of quorum sensing in acyl-homoserine lactoneproducing or-degrading tobacco plants leads to alteration of induced systemic resistance elicited by the rhizobacterium Serratia marcescens $90-166$. Plant Pathol. J. 29, 182-192. doi: 10.5423/PPJ.SI. 11.2012.0173

Schenk, S. T., Stein, E., Kogel, K.-H., and Schikora, A. (2012). Arabidopsis growth and defense are modulated by bacterial quorum sensing molecules. Plant Signal. Behav. 7, 178-181. doi: 10.4161/psb. 18789

Schikora, A., Schenk, S. T., Stein, E., Molitor, A., Zuccaro, A., and Kogel, K.-H. (2011). N-acylhomoserine lactone confers resistance toward biotrophic and hemibiotrophic pathogens via altered activation of AtMPK6. Plant Physiol. 157, 1407-1418. doi: 10.1104/pp.111.180604

Schuhegger, R., Ihring, A., Gantner, S., Bahnweg, G., Knappe, C., Vogg, G., et al. (2006) Induction of systemic resistance in tomato plants by $\mathrm{N}$-acylhomoserine lactone-producing rhizosphere bacteria. Plant Cell Environ. 29, 909-918. doi: 10.1111/j. 1365-3040.2005.01471.x

Scott, R. A., Weil, J., Le, P. T., Williams, P., Fray, R. G., von Bodman, S. B., et al. (2006). Longand short chain plant-produced bacterial $N$-acylhomoserine lactones become components of phyllosphere, rhizosphere, and soil. Mol. Plant-Microbe Interact. 19, 227-239. doi: 10.1094/MPMI-19-0227

Sieper, T., Forczek, S., Matucha, M., Krämer, P., Hartmann, A., and Schröder, P. (2014). N-acylhomoserine lactone uptake and systemic transport in barley rest upon active parts of the plant. New Phytol. 201, 545-555. doi: 10.1111/nph.12519

Teplitski, M., Mathesius, U., and Rumbaugh, K. P. (2011). Perception and degradation of $N$-acylhomoserine lactone quorum sensing signals by mammalian and plant cells. Chem. Rev. 111, 100-116. doi: 10.1021/cr100045m

Turner, T. R., James, E. K., and Poole, P. S. (2013). The plant microbiome. Genome Biol. 14, 209-219. doi: 10.1186/gb-2013-14-6-209

von Rad, U., Klein, I., Dobrev, P. I., Kottova, J., Zazimalova, E., Fekete, A., et al. (2008). The response of Arabidopsis thaliana to $\mathrm{N}$-hexanoylDL-homoserine-lactone, a bacterial quorum sensing molecule produced in the rhizosphere.
Planta 229, 73-85. doi: 10.1007/s00425-0080811-4

Yi, H.-S., Ryu, C.-M., and Heil, M. (2010). Sweet smells prepare plants for future stress-airborne induction of plant disease immunity. Plant Signal. Behav. 5, 528-531. doi: 10.4161/psb.10984

Zamioudis, C., and Pieterse, C. M. J. (2012). Modulation of host immunity by beneficial microbes. Mol. Plant Microbe Interact. 25, 139-150. doi: 10.1094/MPMI-06-11-0179

Zilber-Rosenberg, I., and Rosenberg, E. (2008). Role of microorganisms in the evolution of animals and plants: the hologenome theory of evolution. FEMS Microbiol. Rev. 32, 723-735. doi: 10.1111/j.15746976.2008.00123.x

Conflict of Interest Statement: The authors declare that the research was conducted in the absence of any commercial or financial relationships that could be construed as a potential conflict of interest.

Received: 30 September 2013; accepted: 19 March 2014; published online: 08 April 2014.

Citation: Hartmann A, Rothballer $M$, Hense BA and Schröder P (2014) Bacterial quorum sensing compounds are important modulators of microbe-plant interactions. Front. Plant Sci. 5:131. doi: 10.3389/fpls.2014.00131

This article was submitted to Plant-Microbe Interaction, a section of the journal Frontiers in Plant Science.

Copyright $\odot 2014$ Hartmann, Rothballer, Hense and Schröder. This is an open-access article distributed under the terms of the Creative Commons Attribution License (CC BY). The use, distribution or reproduction in other forums is permitted, provided the original author(s) or licensor are credited and that the original publication in this journal is cited, in accordance with accepted academic practice. No use, distribution or reproduction is permitted which does not comply with these terms. 